\title{
Learning to Hoard: The Effects of Preexisting and Surprise Price-Gouging Regulation During the COVID-19 Pandemic
}

\author{
R. Chakraborti ${ }^{1} \cdot$ G. Roberts $^{2}$
}

Received: 24 August 2020 / Accepted: 21 June 2021 / Published online: 2 August 2021

(C) The Author(s), under exclusive licence to Springer Science+Business Media, LLC, part of Springer Nature 2021

\begin{abstract}
Theory suggests anticipation of shortages stemming from price regulation can motivate households to stock up more and thereby aggravate the regulation-induced shortage. We test this theory on online shopping searches for two typically store-bought staples: hand sanitizer and toilet paper. Combining (i) interstate variation in type of price-gouging regulation - preexisting versus surprise versus none, (ii) their temporally staggered implementation, and (iii) the demand surges for hand sanitizer and toilet paper during the COVID-19 pandemic facilitates identifying the impacts of different price-gouging regulation on consumer searches. Our results are consistent with price-gouging regulationdriven anticipatory hoarding. Difference-in-differences estimates reveal that states with preexisting-regulation experience the largest increases in post-implementation search proportions for both products. Accounting for potential endogeneity of implementation using a nearest-neighbor matching strategy reveals states that make surprise announcements of new regulation during the pandemic also experience larger increases in postactivation hand sanitizer search proportions than states without any such policy, but smaller increases than what preexisting-law states experience. These results corroborate the theoretical predictions about consequences of regulation-induced anticipation of shortages and inform the current policy debate surrounding impacts of price-gouging laws. Fundamentally, our results indicate behavioural responses to policy evolve as experience reveals the effects of the policy, and this evolution might influence the welfare consequences of the policy.
\end{abstract}

Keywords COVID-19 $\cdot$ Price gouging $\cdot$ Shortages $\cdot$ Price controls $\cdot$ Panic buying

\author{
G. Roberts \\ gavinroberts@weber.edu \\ R. Chakraborti \\ rik.chakraborti@cnu.edu
}

Extended author information available on the last page of the article 
Symptoms of widespread "hoarding" and "panic buying"-large queues for staples, shelves upon shelves lying empty at retail outlets, vicious fights breaking out in stores over the precious few remaining hand sanitizer bottles or toilet paper rolls - proliferated daily headlines during the onset of the COVID-19 pandemic in the United States of America (USA) in Spring 2020. This paper explores whether regulations aiming to keep prices down during the pandemic may have contributed to such behaviours. Specifically, we examine whether previous experience with shortages induced by price-gouging regulation-prohibitions on price increases during emergencies - inspired attempts to stock up more during the early stages of the pandemic. If so, our results are of interest to policy makers and scholars studying price-gouging regulation: Regulation-induced anticipated shortages may be self-fulfilling, and the objectives motivating such regulation self-defeating.

While the possibility of experience-driven anticipatory hoarding has these key implications for the general welfare consequences of price-gouging regulation, empirical verification requires comparing behavioural responses of the experienced and the inexperienced to active price-gouging regulation. Opportunities to do so are uncommon: Causal identification requires external variation not only in (i) activation of price-gouging regulation across regions confronted with similar emergencies to isolate the general impacts of such regulation, but also in (ii) type of active price-gouging regulation itself that permits separating experience-driven responses to anticipated consequences of active price restrictions from reactionary responses to actual prices resulting from these active price restrictions. The COVID-19 pandemic's arrival and surge in the USA offers just that rare opportunity.

The pandemic's sudden and rapid spread across the USA triggered a sequential flurry of statewide emergency declarations in March 2020 (see Fig. 2). While these declarations activated preexisting price-gouging regulations in 34 states that had enacted these long before the pandemic's arrival, eight other states enacted and activated new price-gouging regulations through executive orders in response to the growing crisis. The remaining states, on the other hand, declared statewide emergencies, but did not have preexisting price-gouging regulations, neither did they introduce new ones. This variation in price-gouging regulation across a large number of states during the pandemic facilitates an empirical inquiry of behavioural responses to these different types of regulations. Public emergencies like hurricanes that normally trigger preexisting price-gouging regulations tend to be localized, and the emergency-induced scarcity remains isolated to relatively few states. This isolation limits the scope of empirical analysis because suitable counterfactual states - ones that experience similar demand surges driven by the emergency but do not activate price-gouging regulations - are very limited. The interstate price-gouging policy variation triggered by the pandemic, in contrast, allows us to observe the evolution of behavioural responses to price-gouging regulation at three distinct phases: no policy, new surprise policy without the opportunity for anticipation or learning, and longstanding policy with the opportunity for anticipation and learning. We exploit this variation to examine the impacts of preexisting and new price-gouging regulation on online shopping search behaviour.

The official announcements activating price-gouging regulation, preexisting, or surprise, frequently emphasize ensuring availability of essentials at fair prices as their key goal. For instance, Governor Newsom of California, in announcing his executive order banning price gouging, mentioned "ensuring that all consumers are able to purchase what they need, at a fair price" as a key objective (CA.gov 2020). Based on these objectives, regulatory restrictions on price gouging receive widespread popular support on moral grounds (Brewer 2007; Hiltzik 2017; Pan 2020; Snyder 2009). 
Most economists, and some legal scholars, though, continue to warn against the shortageinducing tendencies of these regulations. They emphasize the necessity of price surges to ensure store shelves do not run dry of essentials precisely when they are needed the most (e.g., Niles 2020). Standard economic theory cautions against price-gouging regulations which impose price ceilings on competitive prices and cause shortages if they bind prices below the equilibrium level (Barzel 1974). With prices fixed, search costs adjust to clear markets. This mechanism imposes unproductive costs on households and results in deadweight losses (Platt 2009). While the theoretical debate surrounding the consequences of price-gouging regulation is long standing, empirical evidence on the matter is scant, especially regarding their impacts during the COVID-19 pandemic. To the best of our knowledge, our work is the first to investigate differences in how expected and unexpected activation of price-gouging regulation affects shopping searches.

We expect differences in the impacts between preexisting and new price-gouging regulations on online shopping searches based on three possible anticipatory responses. First, anticipating regulation-induced shortages may motivate households to stock up more in advance (Deacon and Sonstelie 1989; Fleck 2014; Weitzman 1991). When stores do not increase prices in response to growing scarcity, these lower prices subsidize and inspire hoarding behaviour (Hansman et al. 2020). Second, hoarding may also be inspired by anticipated arbitrage opportunities arising from the regulation (Hansman et al. 2020). Past experience with regulation-induced shortages may motivate forward-looking individuals to seek arbitrage opportunities across state lines: stocking up at prices held low by regulation in active-law states, and selling in states without such laws. Third, anticipation of shortages related to price-gouging legislation by some buyers may also exacerbate shortages and panic buying via information cascades and herd behaviour, e.g., buyers stock up staples because they observe other buyers stocking up staples (Bikchandani and Hirshleifer 1992; Huremovic 2019; Sterman and Dogan 2015). In the case of preexisting price-gouging regulations which are triggered as soon as a statewide emergency is declared, households have the opportunity to anticipate shortages and may have learned about shortages resulting from price-gouging regulation through previous experience. These opportunities for learning and anticipation may lead buyers to stock up in advance of actual shortages. On the other hand, in the case of surprise announcements of new price-gouging regulation introduced during the emergency itself, buyers have a smaller opportunity to anticipate resulting shortages, and are less likely to have experienced price-gouging regulation in the past.

We estimate the treatment effects of preexisting and surprise regulation on online shopping searches by interacting state-level variation in price-gouging regulation with the variation in timing of activation. We use a difference-in-differences model and a nearest-neighbor matching model to estimate the effects of different price-gouging policies on consumers' online searches for hand sanitizer and toilet paper, two typically store-bought staples that experienced demand surges due to the pandemic.

Our work is complementary to recent literature exploring the consequences of price rigidity, imposed or otherwise. Beatty et al. (2021), by comparing station-level availability and prices of gasoline in Florida and Louisiana, find little evidence of price-gouging tendencies in stores right before hurricanes, but do find evidence consistent with shortage-inducing tendencies of the price-gouging regulations themselves. Using US supermarket scanner data, Hansman et al. (2020) show that sticky prices during the global rice crisis of 2008 motivated consumer hoarding. Their results indicate that this hoarding response to decoupling of prices from the increased scarcity was motivated primarily by future personal consumption, and richer 
households exhibited stronger tendencies to hoard in response to sticky prices. While Beatty et al.'s (2021) results confirm the shortage-inducing tendencies of price-gouging regulation, Hansman et al.'s (2020) results provide evidence consistent with the mechanism we propose through which price regulation may drive hoarding tendencies.

Our work makes three specific contributions. First, the results offer policy-relevant insights regarding the potential adverse consequences of price-gouging regulation during the pandemic. Barring the exceptions of Cabral and Xu (2020) and Chakraborti and Roberts (2020), no empirical work has addressed this issue. Second, our work takes the empirical literature exploring the consequences of price-gouging regulation forward by identifying a novel channel - anticipatory hoarding - that may aggravate the regulation-induced shortages. Third, the results speak to the general possibility that anticipation of policy consequences may alter behaviour, and thereby the efficacy of the policy.

\section{Context, Theory, and Hypotheses}

The first confirmed case and first instances of community spread of COVID-19 in the USA occurred in January 2020 (Ghinai et al. 2020). The World Health Organization (WHO) declared the COVID-19 outbreak a global pandemic on 11 March 2020 (World Health Organization 2020). Total confirmed cases rose to almost three million in the USA by early July 2020 (John Hopkins University and Medicine 2020). The spread of COVID-19 led to an unsurprising increase in demand for certain household products such as hand sanitizer. However, it also led to surprising shortages for products such as toilet paper which were widely reported in the media (for example, see Gardizy (2020)). Price-gouging regulation may have influenced the occurrence and magnitude of these shortages.

We use Google Shopping Trends to measure search popularity for hand sanitizer and toilet paper - two goods that were known to be in short supply during the COVID-19 pandemic. Shortages of hand sanitizer and toilet paper frequently made news headlines in Spring 2020 during the first wave of the COVID-19 pandemic.

Figure 1 presents the comparisons of the nationwide Google Shopping search trends for hand sanitizer, and toilet paper, our key outcome variables of interest, and pizza, one of the most searched terms nationwide in regular times, from late-January 2020 to early-May 2020 along with some key dates associated with the US COVID-19 pandemic. In this three-product comparison, the product that acquired the highest shopping search volume obtains a score of 100 on the day it achieved this maximum, and the rest of the product-day observation scores reflect relative proportions of this maximum (see the "Main Results" subsection: the data below for more details). We include the national shopping search trends for pizza in the figure to provide a comparative baseline for the extent to which the pandemic's onset instigated shopping searches for hand sanitizer and toilet paper.

On regular days, shopping searches for pizza dwarf shopping searches for hand sanitizer, toilet paper, and other household staples. Figure 1, however, reveals a trend reversal along the timeline considered here: The explosive rise in shopping searches for toilet paper around midMarch dwarfed shopping searches for pizza; shopping searches for hand sanitizer also surged enough to exceed shopping searches for pizza around this time.

Figure 2 shows the distribution of state-level price-gouging regulations during the pandemic. Eight states neither had regulatory restrictions on price gouging before the pandemic nor enacted any in response to the pandemic. Figure 2 displays these "No Law" states in white. 


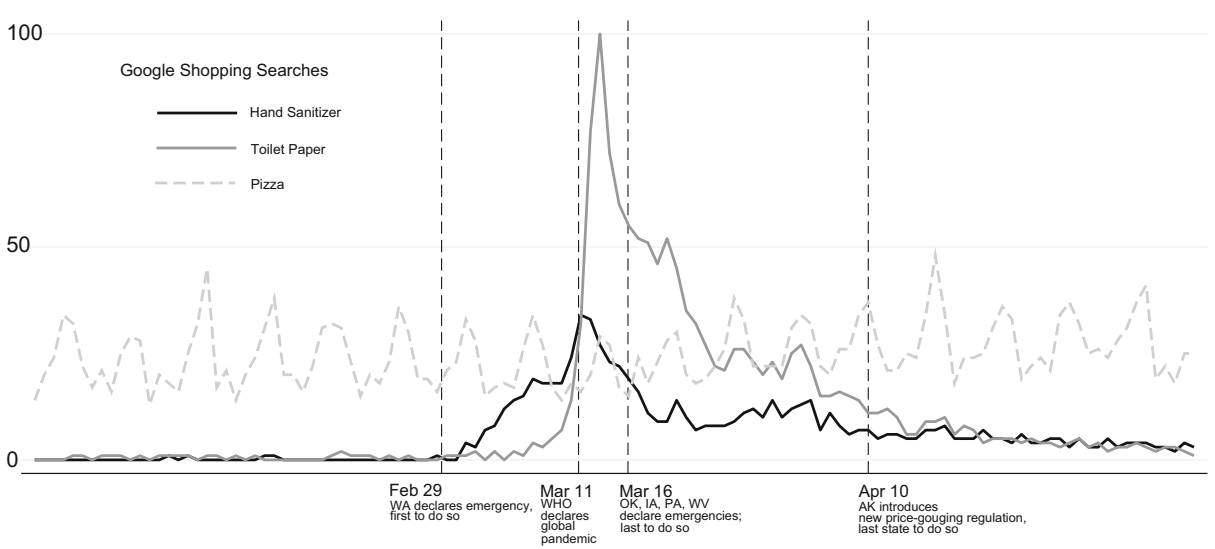

Fig. 1 National shopping search trends and pandemic timeline in the USA. Note: Google Shopping Trends search data indexes relative shopping search volumes on a 0-100 scale. Higher values for a search query reflect higher shopping category searches for a product. A search term scores a value of 100 on the day that it is most popular, relative to all other days, and all other search terms considered. Other scores for all search terms presented reflect daily search popularity relative to search volumes generating that maximum score of 100 . Source: Hand Sanitizer, Toilet Paper, and Pizza Searches on Google Shopping Trends

Figure 2 also presents a timeline showing dates at which states activated price-gouging laws, through declarations of statewide emergencies in case of preexisting laws or through executive orders in case of surprise laws.

Most states had price-gouging regulations on the books for many years before the COVID19 pandemic unfolded, especially those that had experienced large increases in prices during natural disasters in the past. For example, Louisiana last revised its price-gouging regulation in 2010 (Louisiana State Legislature 2020; Tarrant 2015). State-level price-gouging regulations that were on the books prior to the COVID-19 pandemic are generally activated upon implementation of an emergency declaration by the governor of the state (King and Spalding Law Firm 2020). Households in states with preexisting price-gouging laws have an opportunity to anticipate the effects of price-gouging regulation and may have experienced these effects in the past.

Several states that did not already have price-gouging regulation on the books enacted regulations after their COVID-19 emergency declarations. These "Surprise Law" states are shown in gray in Figure 2. Households in these states had less time to anticipate the effects of price-gouging regulations and are less likely to have had past experience regarding their effects. We refer to these unanticipated enactments of price-gouging regulation as "surprise laws" in this article, while we refer to price-gouging regulation in place before the onset of the COVID-19 pandemic as "preexisting laws."

Two states, Massachusetts and Illinois, merit a quick digression. While these states have pre-existing laws prohibiting price gouging, their scope is limited only to petroleum products. But in response to the pandemic, executive orders from the states' governors expanded the scope of these regulations to include hand sanitizer and toilet paper among other essentials. Though experience with gasoline shortages resulting from price-gouging regulation during past emergencies may have spillover effects to other products, we choose to take a conservative approach: We encode these states as ones with surprise laws, and we take the date of the governor's executive order as the date of activation of these laws. We make similar judgement calls in classifying Michigan also as a surprise-law state; see Note (2) under Figure 2. 


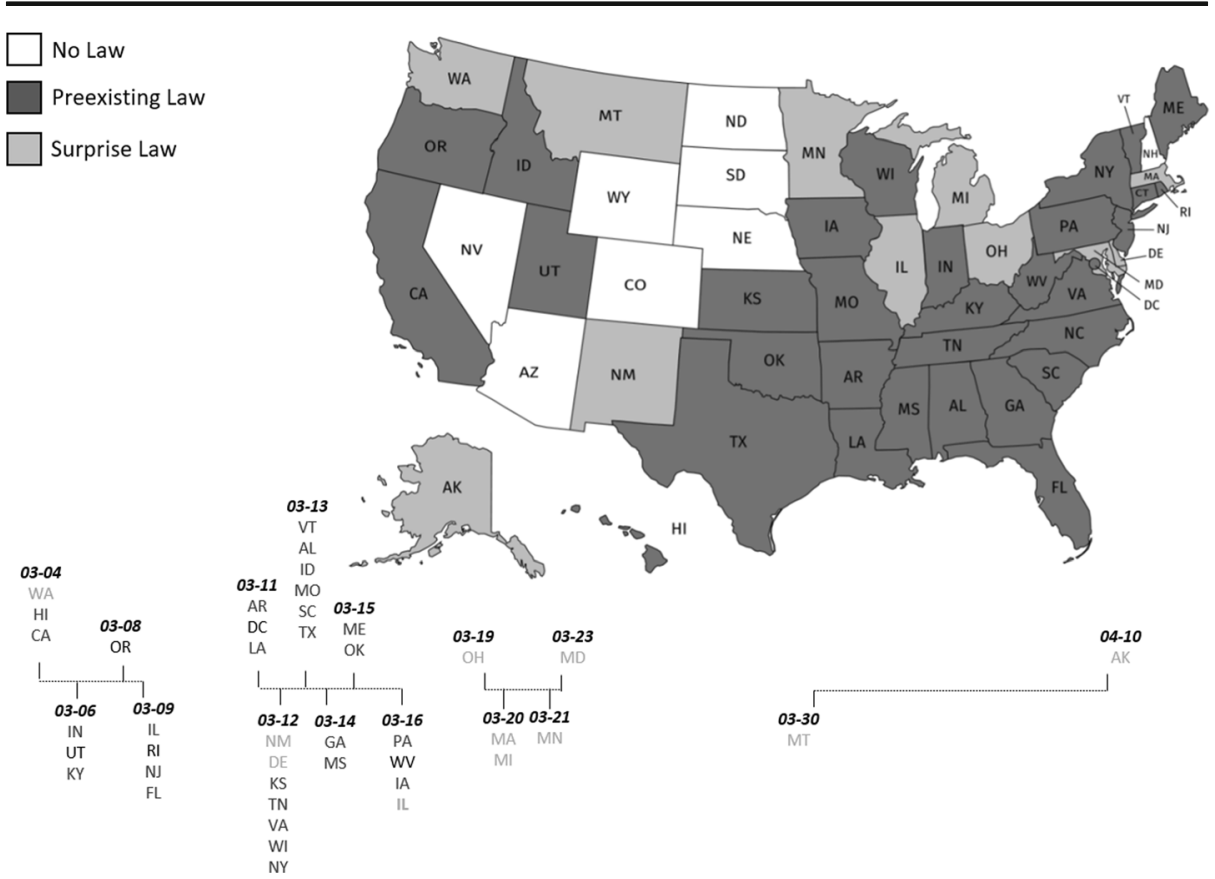

Fig. 2 Statewise distribution of price-gouging laws, and activation dates. Note (1): Though Illinois and Massachusetts have preexisting laws, these apply only to petroleum products. While the pandemic-related statewide emergency declarations activated these laws, both states also activated new "Surprise" laws which expanded the scope of these laws to all pandemic-related essentials including hand sanitizer and toilet paper, through executive orders. Accordingly, we treat these states as "Surprise Law" states, and use the dates of activating these new regulations as the date of these states" "surprise" activation. Note (2): A preexisting and always active consumer protection law in Michigan prohibits selling goods for prices higher than typical for the area, but this law makes no mention of emergencies. However, after declaring the pandemic-related emergency on March 10th, Michigan's governor introduced executive order no. 2020-8 on March 20th, which strengthened the existing law by extending its coverage to products like face masks, hand sanitizer, cleaning supplies, and paper products. We take this March 20th declaration as the date of Michigan's "surprise" activation. Source: The King \& Spalding law firm's COVID-19 2020 Survey of Federal and State Price Gouging Laws

Robustness checks verify this classification is indeed conservative: Consistent with our anticipation-based predictions, the impacts of preexisting laws relative to surprise laws turn out to be stronger if we classify these states instead as preexisting-law states (see Table 1 and Table 2 in Online Appendix A).

The specifics of price-gouging regulation vary across states. For example, some state's policies impose criminal penalties for price gouging while others only allow for civil actions. For the purposes of our analysis, we assume that price-gouging regulations had similar effects on sellers' propensity to increase prices regardless of these technical differences in regulation. Tarrant (2015) provides detailed historical background related to price-gouging laws in states susceptible to hurricanes. Many of these states passed laws in reaction to natural disasters that led to large price increases for certain goods like retail gasoline. A similar trend occurred during the pandemic with the sudden activation of price-gouging regulation in the eleven "Surprise Law" states.

Economic theory predicts that price-gouging regulation could lead to binding price ceilings causing shortages, consumer waiting, and increased consumer search (Barzel 1974; Brewer 2007; Culpepper and Block 2008; Montgomery et al. 2007; Weitzman 1991; Zwolinski 2008). 


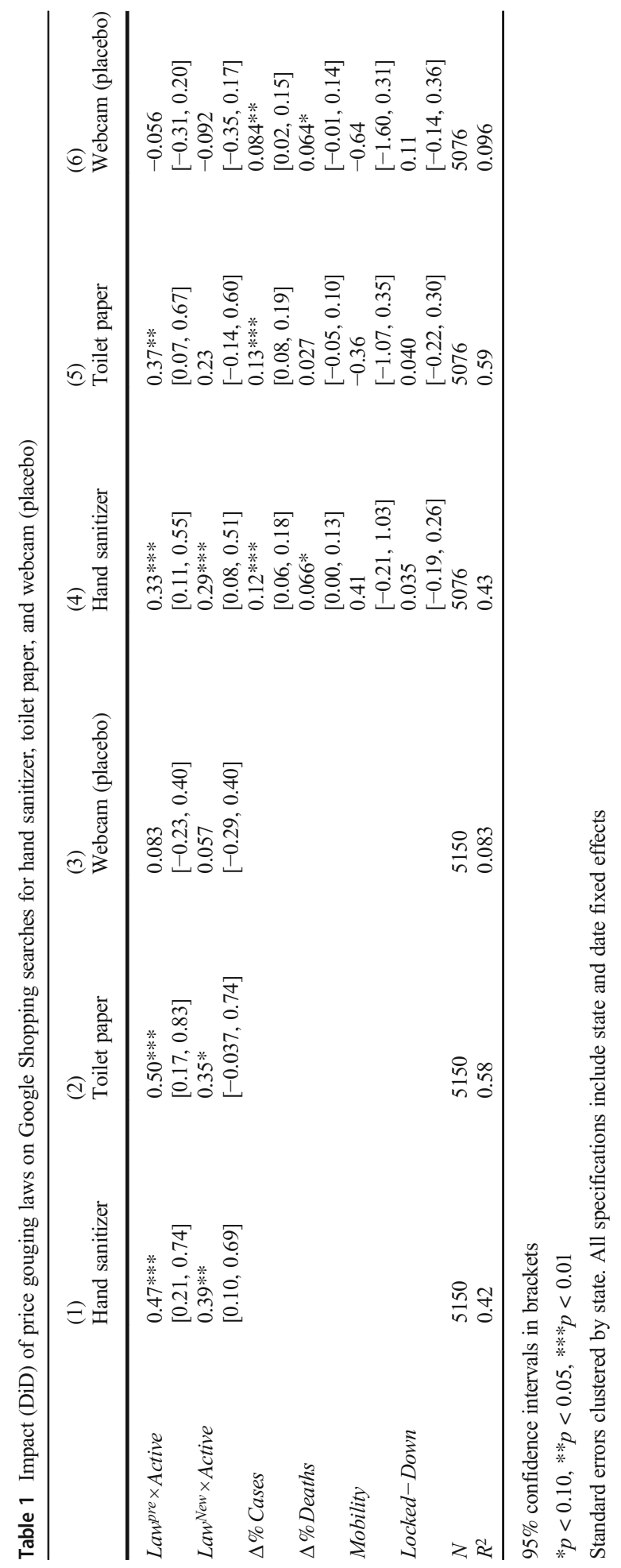




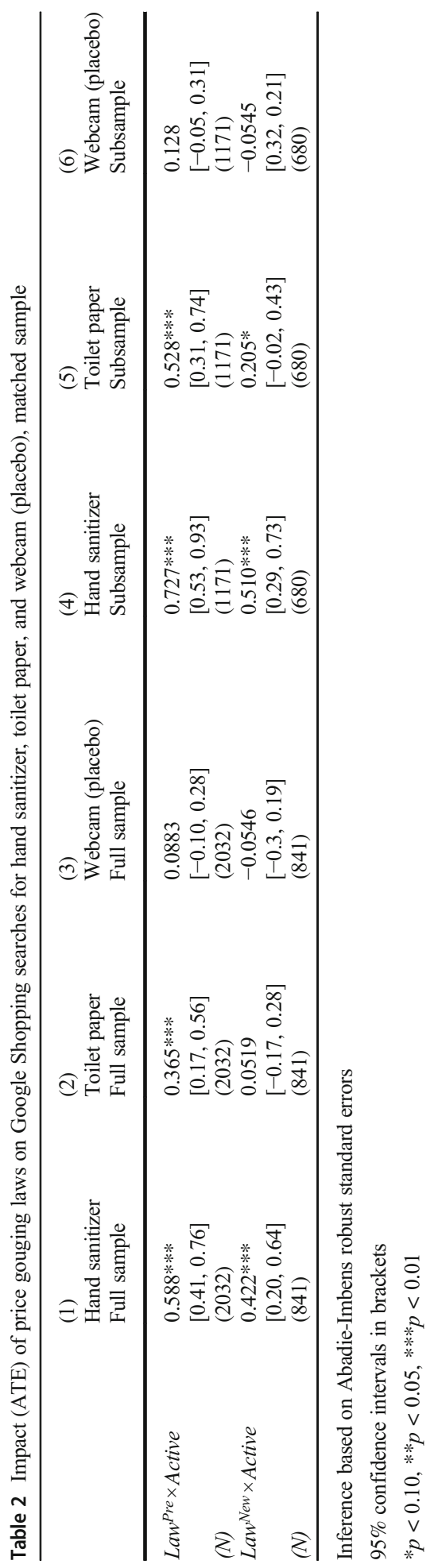


Empirical evidence on the effects of price-gouging regulations is less abundant. Montgomery et al. (2007) estimate that price ceilings resulting from price-gouging regulation would lead economic damages in the two-month period following hurricanes Rita and Katrina to increase by $\$ 1.5-2.9$ billion. Tarrant (2015) finds that price-gouging regulations decreased economic growth after hurricanes but that these states quickly recovered after emergency declarations expired. Beatty et al. (2021) exploit the external variation in timing and location of hurricane landfalls in Florida and Louisiana to study price-gouging tendencies during hurricanes and impacts of price-gouging laws. Using detailed station-level data on retail and wholesale prices, retailer margins, fuel price pass-through, the paper finds no evidence consistent with widespread price gouging. Instead, the paper presents evidence consistent with shortages resulting from the price restrictions imposed by price-gouging laws. Recently, Chakraborti and Roberts (2020) show that Google Shopping Trends were significantly higher in states with pricegouging legislation compared to states without regulation during the early stages of the COVID-19 pandemic using a difference-in-differences analysis. To the best of our knowledge, Cabral and $\mathrm{Xu}$ (2020) is the only other existing study that investigates price gouging in the context of the COVID-19 pandemic. Using consumer reviews as a measure of seller reputation on Amazon, they find evidence reflecting seller reputation has a moderating effect on a seller's propensity to price gouge following a demand shock.

The existence of price-gouging regulation in some states before the pandemic and the sudden enactment and activation during the pandemic in others allows for comparison of the effect of these policies when market participants are and are not surprised by them. If consumers learn about the effects of price-gouging laws through experience, they may behave differently in response to their activation. Weitzman (1991) proposes that "search" or "waiting" costs can lead consumers to adopt a hoarding policy that leads to or exacerbates shortages. Of course, consumers would need to anticipate these costs before implementing a hoarding policy. Exogenous state-level variation in price-gouging policies allows us to compare differences in household search behaviour across states with anticipated pricegouging policies, surprise price-gouging policies, and no price-gouging policies.

Anticipation of hand sanitizer and/or toilet paper shortages and actual shortages are both expected to lead to increased consumer search. We expect consumers to engage in increased online searches in states with price-gouging regulation: Price-gouging regulation should lead to the highest searches in states with preexisting regulation, and the second highest searches in states with surprise regulation, compared to states without regulation.

\section{Methods}

Identifying the causal effect of price-gouging regulation on consumer searches is challenging because of potential endogeneity concerns. First, any observed correlation between pricegouging regulation and consumer searches may be spurious: Both may be driven by a third variable. Second, reverse causation may drive the observed correlation if shortages lead political forces to mobilize in support of price-gouging regulation. Confronted with these challenges, estimating the causal effect of interest hinges on finding an empirical setting in which variation in price-gouging laws arises exogenously. The specific source of exogenous variation we exploit in this paper is the onset of the pandemic-induced state emergency declarations which activated the price-gouging regulation in preexisting-law states, and led to the introduction of new price-gouging regulation in surprise-law states. 


\section{Data}

\section{Series and Sources}

To evaluate the impact of these price-gouging regulations on online shopping search trends, we assemble a panel data set consisting of daily Google Shopping queries on "hand sanitizer," "toilet paper," and "webcam" from all 50 US states. We include webcam shopping searches to facilitate a placebo test across treatment and control groups. Webcams experienced a pandemic-driven surge due to work- and study-from-home situations, but webcams do not meet the criteria for price-gouging regulations. Therefore, online shopping searches for webcams should exhibit similar trends across treatment and control states. Restricting attention only to the "Shopping" category of Google Trends for these goods, rather than all categories, avoids ambiguity: This restriction will filter out, for instance, searches for make-at-home hand sanitizer recipes, or people searching for news related to toilet paper shortages. However, Google's algorithm incorporates search terms related to shopping. For instance, the data on toilet paper search volumes incorporates related search queries like "where to buy toilet paper," "hand sanitizer in stock," and "toilet paper in stock near me."

Our sample range is from 22 January 2020 to 3 May 2020. This range covers the full timeline of the pandemic's initial spread in the USA, from the Centers for Disease Control and Prevention's (CDC) (21 February 2020) announcement that a pandemic was likely to Alaska's activation of new price-gouging regulation on 10 April 2020.

We add information on preexisting price-gouging legislation from the Time Magazine's Summary of State "Price Gouging" Statutes and Regulations, informatio0000000n on new price-gouging regulation from the King \& Spalding law firm's "COVID-19 Survey of Federal and State Price Gouging Laws," and information on dates of statewide emergency declarations from the respective state-government websites.

We then add time- and state-varying pandemic control variables that we suspect may impact online searches including trends in COVID-19 outcomes collected from the COVID19 Data Repository by the Center for Systems Science and Engineering (CSSE) at Johns Hopkins University; we account for the influences of pandemic-induced reductions in mobility on online shopping trends by including cellphone-based intra-state mobility data from Cuebiq, ${ }^{1}$ and information on statewide lock-downs (and re-openings) from The New York Times interactive map. ${ }^{2}$

\section{Outcome Variables}

Google Shopping search values for "hand sanitizer," "toilet paper," and "webcam" constitute our outcome variables of interest. Google Shopping Trends search data indexes relative shopping search volumes on a $0-100$ scale. Higher values for a search query reflect higher shopping category searches in a location compared to the same shopping query on different days in the location. The search term scores a value of 100 on the day that it is most popular in the series. For instance, a value of 50 in North Carolina for "toilet paper" on a given day reflects toilet paper-related shopping queries were half as popular on that day compared to the most popular search day in North Carolina between 22 January and 3 May. Hitting the lower

\footnotetext{
${ }^{1}$ See https://www.cuebiq.com/visitation-insights-covid19/.

${ }^{2}$ See https://www.cuebiq.com/visitation-insights-covid19/.
} 
bound of 0 for any location reflects insignificant search popularity for this term. A key advantage of this measure is its in-built automatic control for larger search volumes in more populated areas and in areas where a product is more or less popular.

\section{Treatment Variables}

We encode preexisting statewide price-gouging legislation as a binary variable ( Law $\left.^{\text {Pre. }}\right)$. We set $L a w^{\text {Pre }}$. equal to one for states which have preexisting price-gouging legislation, and zero for those without any such legislation. We encode surprise statewide price-gouging regulation-those activated in response to the current emergency through state executive order-also as a binary variable $\left(\mathrm{Law}^{\mathrm{New}}\right.$ ). We set $L a w^{\mathrm{New}}$. equal to one for states which introduce surprise price-gouging legislation, and zero for those without any such legislation. Both variables take the value of zero for the control states which neither had preexisting legislation, nor introduced new legislation during the pandemic.

To separate between pre- and post-treatment phases, we encode activation of these regulations as a binary variable (Active). Active is equal to zero for a state prior to its activation of price-gouging regulation, and equal to one thereafter. Active is equal to zero for all days before the surprise-law states activate new regulation, and equal to one thereafter. For no-law states, our control states that neither have preexisting price-gouging regulations nor introduce new ones, we proxy for the post-treatment phase by their post emergency-declaration phase.

\section{Controls}

The pandemic-related variables-logged differences in COVID-19 cases and deaths, the intrastate mobility variable, and the state-lock-down variable - constitute our set of control variables. COVID-19 cases/deaths reflect the severity of the pandemic which may influence a consumer's inclination to engage in online shopping to avoid risking an infection. The intrastate travel variable accounts for the substitutability between searches in physical and online stores. If consumers drive around store to store looking for hand sanitizer, and find enough, shopping online for more hand sanitizer may be unnecessary. However, if these in-store searches reveal depleted stocks in stores, these consumers may start searching more for these supplies online.

To permit interpretation of the estimated impacts as percent changes, we apply a natural log transformation to all our non-binary continuous variables. This transformation also benefits estimation by reducing influences of outlying observations.

\section{Estimation Strategy}

The pandemic's sudden arrival and rapid spread triggered statewide emergency declarations. Published guidelines from organizations including the CDC and WHO led to a surge in hand sanitizer demand. Once states issued lock-downs and stay-at-home orders, spending more time home than at work led to a surge in household demand for toilet paper. The statewide emergency declarations activated preexisting price-gouging regulations, and most states that introduced surprise price-gouging regulations did so as a part of their emergency declarations. Others' surprise regulations closely followed the emergency declarations. For example, Washington, the first state to be affected by the pandemic, declared a statewide emergency on 29 February 2020 and introduced regulations targeting price gouging on 4 March 2020 
while New Mexico declared a statewide emergency on 11 March 2020 and introduced pricegouging regulation the next day.

Our empirical strategy exploits the pandemic-induced statewide shocks to price-gouging regulation to identify the impacts of activating preexisting and surprise price-gouging regulation on consumer searches. As Figure 2 shows, the pandemic's swift spread triggered preexisting laws into action as soon as statewide emergencies were declared in 34 states. Most of these states declared emergencies within a one-week interval around 11 March 2020, the date the WHO declared COVID-19 as a global pandemic. Most of the surprise-law states also introduced their price-gouging regulations around the same time. Crucial to our strategy, because they predate the pandemic, the preexisting laws could not influence the pandemic's spatio-temporal dispersion, which in turn triggered the emergency declarations and activated the regulations.

A naive comparison of post implementation search trends across states under different price-gouging regulation would mistake variation driven by unobserved preexisting differences as variation attributable to differences in price-gouging regulation (Fredriksson and de Oliveira 2019; Lechner 2011). For instance, if average households have more members in states with preexisting regulation which causes a larger proportion of searches for hand sanitizer, the naive approach would end up attributing this increase in relative search volumes to the difference in regulation.

Another naive alternative would compare the difference between pre- and postimplementation phases only in states that implement price-gouging regulation. This approach would mistake variation driven by other changes that occurred at the same time as the policy was implemented as variation attributable to differences in price-gouging regulation (Fredriksson and de Oliveira 2019; Lechner 2011). For instance, suppose WHO's declaration of COVID-19 as a global pandemic coincides with some of state-level emergency declarations that activate price-gouging regulation. Though a part of resulting spikes in hand sanitizer search proportions may result from households responding to WHO's declaration, this naive approach would instead attribute this spike to the difference in regulation as well.

We adopt a difference-in-differences strategy (DiD), which combines the two naive approaches, and avoids the pitfalls of both (Lechner 2011). The DiD approach compares differences in pre- and post-implementation search trends across states with and without pricegouging regulation, surprise, or preexisting. The DiD model explicitly accounts for the impact of preexisting differences in searches, and other changes that coincide with regulatory changes. For example, so long as the difference in state-level demographics that affect consumer searches across the types of states we compare remains stable in the pre- and postimplementation phases, the influence of this difference is eliminated by comparing the within-state difference between the pre- and post-implementation phases. And so long as national shocks that impact consumer searches have a similar impact across all states, their influence is eliminated by comparing the between-state difference across states with and without regulation.

Our DiD specification is

$$
\text { Search }_{s, d}=\alpha_{s}+\delta_{d}+\pi_{1}\left(\text { Law }_{s}^{\text {Pre. }} \times \text { Active }_{s, d}\right)+\pi_{2}\left(\text { Law }_{s}^{\text {New. }} \times \text { Active }_{s, d}\right)+\epsilon_{s, d} .
$$

The dependent variable, Search,$d$, is the log of the Google Shopping trend measure of the product of interest in state $s$ on day $d$. The time-invariant fixed effect $\alpha_{s}$ captures all unobserved state-specific heterogeneous influences that are time invariant, and the state- 
invariant fixed effect $\delta_{d}$ captures the effect of all unobserved nationwide daily variations that could potentially coincide with activation of price-gouging regulation and bias our estimates if unaccounted for.

The coefficient $\pi_{1}$ is the difference-in-difference estimate of the effect of an active preexisting price-gouging law. Similarly, $\pi_{2}$ is the difference-in-differences estimate of the effect of an active surprise price-gouging law. Finally, $\epsilon_{s, d}$. is a mean zero error term.

We also estimate Eq. (1) with additional control variables that may affect the intertemporal and interspatial variation of online searches. Increases in COVID-19 cases and deaths might lead individuals to stay at home and shop online more often so we include state-level logged differences of COVID-19 cases and deaths to control for this possibility. Similarly, some states issued "lock-down" or "stay-at-home" orders during our sample period which may have increased online shopping searches, so we include a variable that is equal to 1 if state $s$ is locked down on day $d$ and is otherwise 0. Finally, we attempt to directly control for individuals' propensity to travel outside their residences by including a state-level daily mobility measure. We assume other state-varying factors that are correlated with searches are time invariant and that other time-varying factors that are correlated with searches are constant across states, so such factors are picked up by the state and date effects in Eq. (1).

Given our design, causal interpretation of the DiD estimates hinges on differences in search trends between groups prior to the activation of price-gouging laws continuing in absence of the intervention. ${ }^{3}$ One way we test this assumption is by applying our DiD regressions to webcams, a good that experienced a demand surge during the first stage of the pandemic but was not subject to price-gouging regulations. Finding a positive treatment effect for webcams would indicate a violation of the parallel trends assumption, and indicate that something other than price-gouging regulation is driving any positive results associated with goods that are subject to such regulation.

If activation of price-gouging regulation is driven by the pandemic's trajectory, and is independent of consumer behaviour, exogeneity of treatment assignment is a safe assumption, and our DiD strategy provides consistent estimates. Such exogeneity, however, may be questioned for states that introduce surprise price-gouging regulation. If some states introduce new regulation in response to growing concerns about forthcoming shortages arising from pandemic-induced hoarding, or price-gouging, the assumption of exogenous treatment assignment for these states is no longer valid. To adjust for this possibility, we also employ the Abadie and Imbens (2011) bias-corrected non-parametric nearest-neighbor matched-sample estimator. The key difference between the DiD estimation strategy and the matched-sample treatment effect estimation strategy arises from the role of covariates. While the DiD strategy accounts for the impact of covariates on the outcome variable, the matching strategy accounts for the role of covariates on treatment assignment itself (Abadie et al. 2004). The matching strategy accounts for the variation in probability of receiving treatment by finding close matches along covariates that influence this probability. Suppose, for instance, a faster rise in COVID-19 cases and deaths influences a state's decision to enact a new price-gouging law to curb anticipated hoarding. The activation of surprise regulation in this case is no longer exogenous. To get around this issue, an appropriate matching strategy first locates close matches between treated and untreated units by changes in cases and deaths so that each matched pair have a similar chance of receiving treatment (activating new laws), and then calculates the average difference in outcomes between all such matched pairs (Abadie et al. 2004; Imbens 2004).

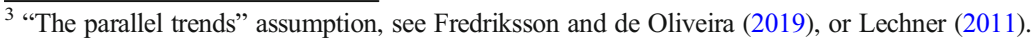


In our context, this strategy involves matching states using specific covariates that may affect the probability of price-gouging regulation enactment and activation. Accordingly, we match on average pre-activation shopping searches and average pre-activation COVID-19 outcomes, the two groups of factors most likely to trigger emergency declarations and/or surprise enactment and activation of price-gouging regulation. Matching on average preactivation search trends has the added benefit of facilitating comparisons only between states with similar average search levels prior to activation. In this matching strategy, we also account for variation in exact treatment duration by forcing exact matches on the number of days since activation; and we account for correlation between physical searches and online searches by including mobility as the final matching covariate. As a final step, after implementing this matching strategy, we visually inspect trends in pre-activation searches between each treatment and matched control pair to identify any concerning differences. Dropping the potentially problematic matches leaves us with a subset of high-quality matches. Charts comparing trends in treatment states with their matched control states are provided in Online Appendix B. We report matched results for both the full sample, and the high-quality subsample.

Consistently estimating causal impacts under these strategies hinges on one final key restriction: No meaningful compositional changes, or spillovers, are allowed (Duflo et al. 2008; Fredriksson and de Oliveira 2019; Greene 2017; Lechner 2011). The search behaviour of consumers in states with a particular type of price-gouging regulation must not influence the search behaviour of consumers in states with another type of price-gouging regulation, e.g., search behaviour in preexisting-law states must be independent of search behaviour in surprise-law or no-law states.

Two potential concerns arise. First, if households in states with active price-gouging regulations anticipate or face shortages, they might travel to neighboring no-law states and search for products there. Though these higher searches would be recorded in no-law states, they are caused by activation of price-gouging regulation in preexisting or surprise-law states. These spillover effects are driven by rational beliefs, and can be a result of past experience with regulation-induced shortages. Second, if households in no-law states expect or observe price increases in their states, and expect active price-gouging laws to keep prices low in neighboring states with active regulation, they may travel to these states and search for products there. Though these higher searches would be recorded in preexisting or new-law states, they would be caused by increasing prices in no-law states.

We cannot directly observe or control for these spillover effects. The first type of spillovers increases searches in no-law states, so it would bias the estimated impacts of price-gouging regulation downwards. The second possibility is more concerning for our estimation strategy since our estimated impacts of preexisting price-gouging regulation would be biased upwards. The rationality underlying the beliefs that drive this type of spillovers, however, is questionable: Households would find such behaviour rational only if price-gouging regulation effectively restricts price increases, but does not create shortages. If they do lead to shortages, consistent with economic principles and past evidence (e.g., Brewer (2007); Culpepper and Block (2008); Deacon and Sonstelie (1989); Montgomery et al. (2007)), then paying the higher price and acquiring the products from local stores in no-law states is likely less costly than traveling to neighboring states only to find empty shelves for goods like hand sanitizer and toilet paper. Furthermore, the voluntary or mandated reduction in mobility arising from infection risks during the pandemic makes travel-driven spillovers to any meaningful extent even more unlikely. While households in some local communities may still hold these beliefs 
and act accordingly, our estimates will be consistent so long as the spillovers are not meaningfully large. We discuss a few other specific concerns and our responses in the "Robustness Checks and Caveats" subsection, after presenting the main results in the following section.

\section{Results}

\section{Main Results}

Figure 3 displays five-day moving average logged search trends. The horizontal axis represents the number of days relative to activation of price-gouging regulation. Search trends in our three state types, pre-existing, surprise, and control, appear very similar in the pre-treatment period. Searches for hand sanitizer begin to increase on average in all state types before emergency declarations occur with control states experiencing increases in sanitizer searches slightly sooner than the treatment states. On the other hand, toilet paper searches do not begin to increase until after price-gouging regulation activation. This figure visually affirms no significant deviations in pre-activation search trends across treatment and control states; in so far as the increase in hand sanitizer searches in control states is problematic, our matching strategy, discussed in detail below, limits the comparisons to treatment-control pairs with similar pre-activation searches.

In the post-treatment period, all three series indicate spiking searches followed by slow declines. Searches for hand sanitizer and toilet paper in pre-existing-regulation states are higher
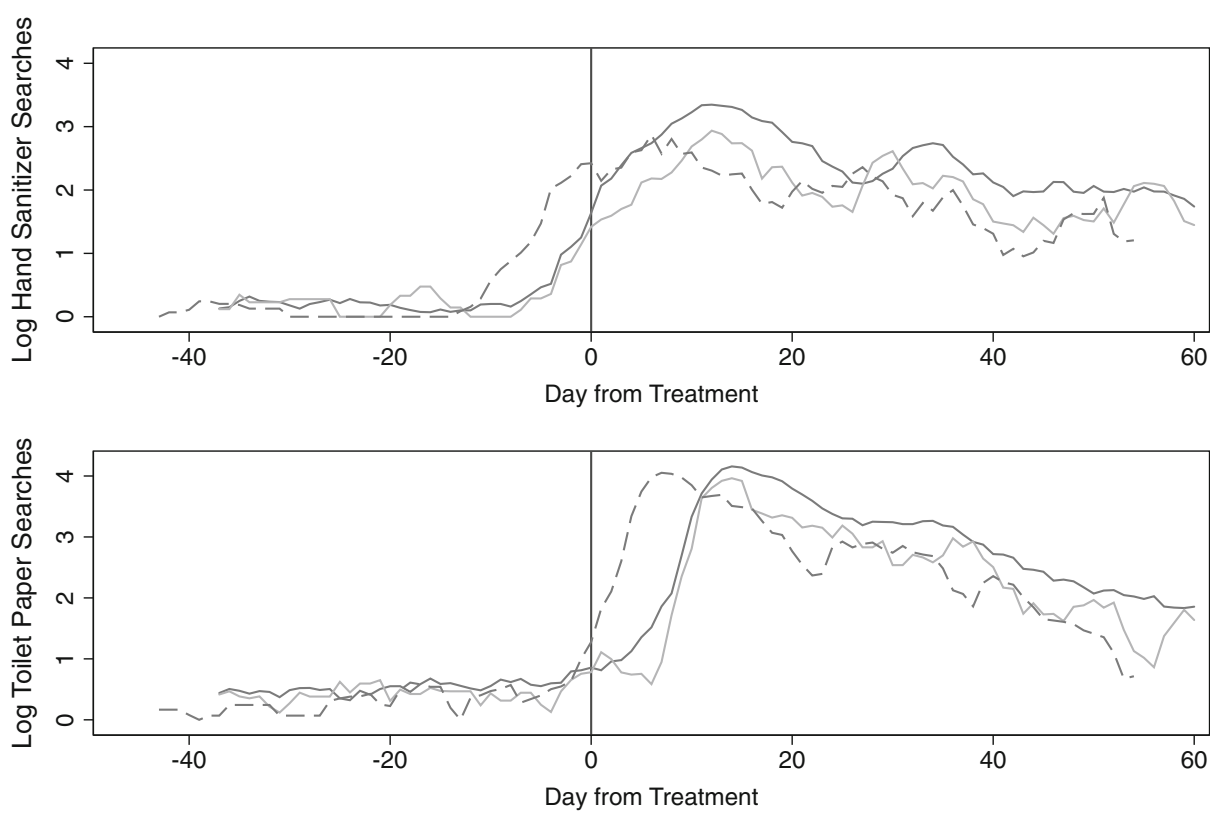

Pre Law

Surprise Law

Control

Fig. 3 Log shopping search trends in pre-existing law, surprise law, and control states 
on average than searches in control states especially after the initial spikes in searches. Searches in surprise-law states also appear larger than average searches in control states in the post-treatment period but the difference is not as clear as it is for pre-existing-law states. Overall, examination of the pre-trends in Figure 3 indicates that these series are amenable to our DiD analysis, that price-gouging regulation led to higher search in price-gouging regulation states, and this effect was larger in pre-existing-regulation states.

Table 1 presents our DiD results. The first column of Table 1 shows the results of estimating Eq. (1) with logged "hand sanitizer" online searches as dependent variable and without pandemic-related control variables. The statistically significant coefficient of 0.47 on the Law $_{s}^{\text {Pre. }} \times$ Active $_{s, d}$ variable indicates that price-gouging regulation increased the $\log$ of daily online hand sanitizer searches in states with preexisting laws by 0.47 , approximately a $60 \%$ increase on average $\left(\mathrm{e}^{0.47}-1=0.60\right)$. The second row of the first column shows that the point estimate in states with surprise laws is 0.39 , and that estimate is significant at the $5 \%$ level. The second column of Table 1 shows the results with logged toilet paper searches as the dependent variable. The estimated effects of price-gouging regulation on logged toilet paper searches are similar to those for hand sanitizer with statistically significant increases in preexisting-law states, and smaller increases in surprise-law states. The third column shows the results of applying our DiD strategy to "webcam" shopping searches. Both treatment effect estimates for webcam searches are small in magnitude and statistically insignificant reflecting similar changes in trends of webcam shopping searches between treatment and control states.

The third, fourth, and fifth columns of Table 1 show the analogous set of results for specifications that include pandemic-related control variables. The estimated impact of price-gouging regulation on logged sanitizer searches in preexisting-law states is 0.33 and statistically significant at the $1 \%$ level. The estimated impact in surprise-law states is also smaller but is now significant at the $1 \%$ level. The estimated treatment effects associated with toilet paper searches are slightly smaller. The estimated treatment effect in pre-existing-law states is 0.37 , significant at the $5 \%$ level, while the treatment effect in surprise states is 0.23 , and not statistically significant.

The changes in the treatment effect estimates upon inclusion of the pandemic-related control variables may indicate an endogeneity problem. Also, the small deviation in hand sanitizer search trends in control states relative to price-gouging regulation states before emergency-declaration activation observed in Figure 3 may be biasing our DiD results for hand sanitizer searches. Recall that our matching strategies account for the potential endogeneity and potential violation of parallel trends by matching on variables predictive of the probability of activation, namely pre-activation searches and COVID-19 outcomes.

Table 2 presents the results of our nearest-neighbor matching strategy. These results suggest similar impacts compared to those estimated from the DiD model. The first three columns provide matched estimates from the full sample. The first row in Column 1 shows that logged online hand sanitizer searches increased by 0.588 in preexisting-law states relative to control states in the post-activation period: Preexisting price-gouging regulation caused an $80 \%$ jump in average daily online searches for hand sanitizer relative to states without price-gouging regulation. The second row in Column 1 shows that logged online hand sanitizer searches increased by about $53 \%$ in states with surprise price-gouging regulation relative to control states. Both results are significant at the $1 \%$ level. The first row in Column 2 indicates that logged toilet paper searches increased by 0.36 in pre-existing-law states and this estimate is also significant at the $1 \%$ level. However, the second row in Column 2 shows that the treatment effect estimate on toilet paper searches for surprise-law states is insignificant in 
the full sample for the matching strategy. The treatment effect on webcam searches, Column 2, both rows, is insignificant reflecting similarity of placebo search volumes across treatment and control groups, which is reassuring.

Columns 4 through 6 in Table 2 display the results of our matching strategy applied to the refined subsample based on visual inspection of pre-trend charts between treatment states and their corresponding control-state matches (see Online Appendix B: Matched Samples and PreTrends). Our treatment effect estimates increase to a small degree in this subsample. Logged hand sanitizer searches and logged toilet paper searches increase by 0.727 and 0.528 in preexisting-law states, respectively, relative to control states. These estimates are both significant at the $1 \%$ level. The estimates in surprise-law states for logged sanitizer and logged toilet paper searches increase to 0.510 and 0.205 , and the latter estimate becomes statistically significant at the $10 \%$ level when the matching strategy is applied to the subsample. Webcam searches reassuringly indicate no significant differences across treatment and control states in this sample as well.

Collectively, our analysis provides strong evidence indicating that activation of preexisting price-gouging regulation increased online searches for hand sanitizer and toilet paper during the COVID-19 pandemic, and activation of new surprise price-gouging regulation increased online searches for hand sanitizer. The larger estimated effects in preexisting-law states compared to surprise-law states are consistent with anticipation of shortages causing or exacerbating shortages and search.

\section{Robustness Checks and Caveats}

This section discusses the variety of robustness checks we performed to verify the consistency of our results, and to reflect upon alternative explanations for our results. We validated the samples with a placebo test, checked for influence of political leaning in driving both the inclination for searches and activation of price-gouging regulation, and confirmed the estimated differences are not purely driven by only duration of treatment exposure. We discuss each of these in turn below.

First, we applied all our main specifications to webcam searches as a placebo test. The results of these regressions presented in Table 1 and Table 2 are encouraging as the treatment effect estimates are all statistically indistinguishable from zero. However, we did observe a statistically significant increase in webcam searches when we applied our matching strategy to a subsample of states with Republican governors, discussed below.

Second, we ran our main specifications on the subsample of states with Republican governors. There are a variety of ways that state-level political variation may be correlated with activation of price-gouging regulation and/or correlated with consumer behaviour. For example, political leaning may be correlated with demand for hand sanitizer across US states and may also impact the probability of activating price-gouging regulations. The results of our $\mathrm{DiD}$ and matching strategies applied to the Republican governor subsample are available in Table A5 and Table A6 in Online Appendix A: Robustness Checks. The DiD results are consistent with the full sample results. The primary difference is that the hand sanitizer treatment effect estimate for pre-existing-law states is statistically insignificant, and the hand sanitizer treatment effect in surprise-law states is only significant at the $10 \%$ level. While these changes partially reflect the decrease in sample size, they could also reflect a problem with our identification strategy: Republican governors are less likely to enact price-gouging regulation while individuals in those states are less likely to search for hand sanitizer. The matching 
results are consistent between the Republican governor states and our full sample for preexisting-regulation states. However, the results for surprise-regulation states reveal a key concern: A large and statistically significant effect for webcam searches, along with a large significant effect for hand sanitizer, and no significant effect for toilet paper, though a coefficient larger than the full sample. This statistically large difference between webcam searches between the control and treatment states is problematic as it indicates a possible upward bias in the estimates for hand sanitizer and toilet paper. Adjusting for this bias would make the coefficient consistent with the earlier results. We identify a few factors that might be driving the bias in the Republican governor subsample discussed below.

The set of Republican governor states that implemented emergency price-gouging regulation is Alaska, Maryland, Massachusetts, Ohio, and Montana. However, we identify Maryland, Ohio, and Montana as bad matches due to concerns about differences in pre-treatment search volumes in those states relative to their control matches (see pre-trend charts for surprise-law states in Online Appendix B: Matched Samples and Pre-Trends): The Republican governor subsample overlaps with the concerning matches we identify in the surprise-law states to a large degree. Furthermore, the control sample becomes more rural in the Republican governor subsample while the surprise-law treatment sample becomes more urban. While these statelevel time-invariant differences are controlled for by the state-level fixed effects in the DiD model, they are not in the matching estimates. However, there could still be a problem if these differences are correlated with the probability of treatment assignment.

More broadly, the presence of a Republican governor may not be a great measure of political variation across states. Most of the surprise-law states with Democrat governors are not "deep blue." For example, Michigan, New Mexico, and Illinois had Republican governors until 2019 and gubernatorial races tend to be competitive between Republican and Democrat candidates in those states. Furthermore, some of the states in our Republican governor sample are considered more "blue" than their Democratic governor counterparts if a wider array of political outcomes is considered. For example, New Mexico had a Democratic governor during our sample period but is to the right of Maryland and Massachusetts on the political spectrum all things considered. The similarity in results across the DiD specifications is reassuring, but it is difficult to disentangle the role of political variation across states given the limitations of our state-level date and we encourage future research to focus more on this issue.

Third, a natural concern arises about whether activation of new laws was less predictive of search because new laws were activated later than preexisting laws on average in our sample. Under normal circumstances, enactment of new regulations may take lawmakers weeks; if so, the possibility that the shortages of hand sanitizer and toilet paper subside by the time the newly enacted law is activated cannot be ignored. This mechanism would lead to an underestimation of the true effects of activation of new laws. However, given the pandemic's rapid surge across the USA, surprise price-gouging regulations in our context were enacted and activated by the same executive order from the governor, which implies no delay between enactment and activation. Our specific robustness checks in Table 3 and Table 4 address this concern by using March 15th as the activation date across all states. The results stay consistent with our main findings indicating that these differences are not explained by differences in activation timing. We also checked using March 10th (Tables A7 and A8 in Online Appendix A) and March 20th (Tables A9 and A10 in Online Appendix A) as earlier and later activation dates. These alternative specifications produce results consistent with the main specifications. The only minor change is under the March 20th activation date, the DiD treatment effect of surprise laws on hand sanitizer is slightly larger than that of preexisting laws. 


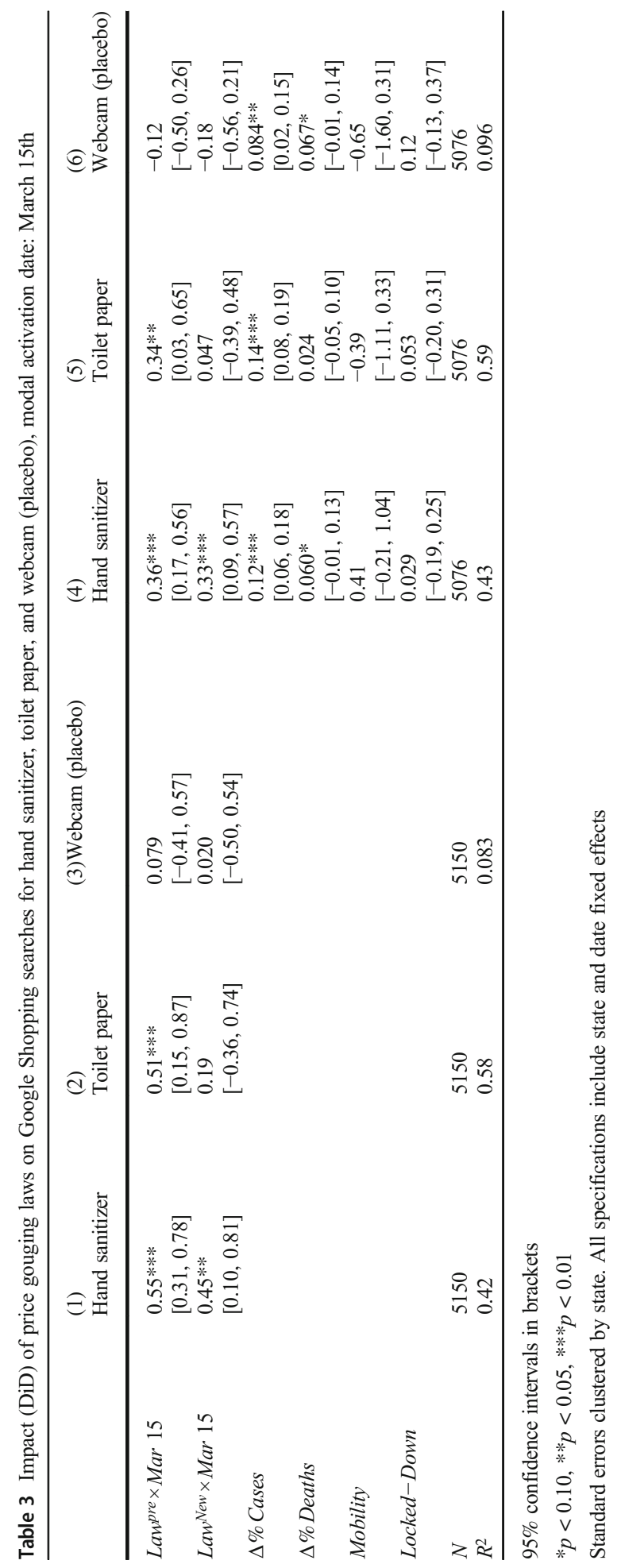


Table 4 Impact (ATE) of price gouging laws on Google Shopping searches for hand sanitizer, toilet paper, and webcam (placebo), modal activation date: March 15th

\begin{tabular}{llll}
\hline & $(1)$ & $(2)$ & $(3)$ \\
& Hand sanitizer & Toilet paper & \begin{tabular}{l} 
Webcam (placebo) \\
\hline Law
\end{tabular} \\
& $0.527^{\text {Pre }} \times$ March 15 & $0.409^{* * *}$ & 0.0697 \\
$(\mathrm{~N})$ & {$[0.35,0.71]$} & {$[0.21,0.60]$} & {$[-0.11,0.25]$} \\
Law ${ }^{\text {New }} \times$ March 15 & $(2072)$ & $(2072)$ & $(2072)$ \\
& $0.304 * * *$ & $0.377 * * *$ & 0.0336 \\
$(\mathrm{~N})$ & {$[0.089,0.52]$} & {$[0.15,0.61]$} & {$[-0.19,0.26]$} \\
\hline
\end{tabular}

Inference based on Abadie-Imbens robust standard errors

95\% confidence intervals in brackets

$* p<0.10, * * p<0.05, * * * p<0.01$

The fourth concern is that increases in searches are driven by attempts to enforce active price-gouging regulation rather than by shortages, actual or anticipated. For instance, enforcement officials could be attempting to monitor prices. However, the comparison of search trends between hand sanitizer, toilet paper, and pizza in Figure 1 indicates that the scale of change is unlikely to have been driven by enforcement searches alone. Furthermore, review of price-gouging regulations across states also reveals that the primary mechanism of enforcement is via a hotline dedicated to consumer complaints. We searched the internet for news stories related to specific instances of price-gouging enforcement, and the vast majority of the examples arise from consumer complaints. We found only one example that indicated prosecutors used online posted prices as evidence in a price-gouging case at trial. If widespread though, this problem would lead to an upward bias in our treatment effect estimates. Separating enforcement-driven searches from shortage-driven searches may be a promising avenue for future work.

Our final concern relates to the possibility that shortages resulting from price-gouging regulation in some states led to more searches in control states. For example, if individuals in control states watched news stories discussing nationwide shortages of toilet paper, this might have led them to stock up on toilet paper. Such news stories should have had similar impacts in treatment states in which case the DiD methodology controls for such differences. If such news stories spur more searches in regulation states because shortages are larger in those states, the effect is consistent with our theory. However, if such news stories have a larger impact in control states, our results would be biased downwards by such a spillover effect. If so, our estimates can be interpreted as lower bounds of the true magnitudes.

In summary, there are important potential confounding factors that should be considered when interpreting our results. Overall, our results appear to be consistent with the theory that price-gouging regulation causes shortages, and that these shortages are exacerbated by anticipatory behaviour. However, assessing the impacts of these potential confounding factors could be a promising avenue for future research.

\section{Discussion and Conclusion}

The COVID-19 pandemic's sudden emergence and rapid spread in the USA activated preexisting price-gouging regulation in many states and led to the enactment of new price- 
gouging regulations in other states. This spatial and temporal variation provides a unique opportunity to examine the impacts of preexisting and new price-gouging regulation, which we exploit in this study. Our results provide evidence that states' activation of preexisting price-gouging regulation, and introduction of new price-gouging regulation both caused households to increase online searches for the consumer staples of hand sanitizer and toilet paper during the 2020 COVID-19 pandemic in the USA, but activation of preexisting regulation has a larger effect. This finding highlights the possibility that learning about, and anticipation of, shortages induces households to hoard goods.

Our results are consistent with standard microeconomic theory and extensions elaborating the role of anticipation in driving household responses to price regulation. If price-gouging regulations bind during a public emergency, then they will cause shortages leading consumers to invest more in search for products (Barzel 1974). Statistically and economically significant estimated impacts for both preexisting and new price-gouging regulation for hand sanitizer support this conclusion. Consumers who have the opportunity to anticipate potential shortages caused by price-gouging regulation may engage in behaviour that exacerbates or even causes shortages such as hoarding and panic buying (Deacon and Sonstelie 1989; Weitzman 1991). The larger estimated impacts of price-gouging regulation on online hand sanitizer and toilet paper shopping searches in states with preexisting price-gouging regulation than in states with new surprise price-gouging regulation during the pandemic support these anticipatory behavioural effects subject to the caveats discussed in the previous section.

Our results indicate that individuals learn about and anticipate the effects of regulation. Changes in behaviour resulting from this learning and anticipation may lead to unintended policy effects. Welfare analysis of price-gouging policies will be inaccurate if they do not account for the effects of learning about the consequences of price-gouging regulation and the opportunity to anticipate these consequences. In this case, we expect that standard welfare analysis would underestimate the costs of price-gouging regulation due to the increased deadweight loss resulting from the compounding of shortages, and from increased consumer investment in search. Learning and anticipation could affect welfare analysis in other policy contexts as well and could increase the benefits of new policies in some cases. Future research should seek out other opportunities to exploit variation in consumer experience with policy to explore the significance of such experience in other contexts.

Our analysis suggests several other immediate avenues for further work. Our anticipatory hoarding hypothesis is founded on micro-level motives of future consumption, reselling, or herding. But our data limits our empirical test to the state level. Our results are consistent with price-gouging regulation-driven anticipatory hoarding, but data limitations impede the scope of a deeper exploration of the extents to which future consumption, arbitrage, or herding mechanisms drive these anticipatory hoarding responses. Exploring whether the differences we observe persist in consumer searches at a higher level of granularity, e.g., counties/cities/census blocks/stores, would be a promising avenue for future work. More granular transaction-level data would be useful in addressing these, and many of the issues discussed in the "Robustness Checks and Caveats" subsection as well especially related to the role of political variation across states. Differences in the seriousness of price-gouging as an offence (civil versus criminal), and associated penalty, vary by state. Future work should also explore the differential impact of severity of punishments and stringency of the laws. Finally, given the significant welfare consequences, and paucity of empirical evidence on the topic, future research should continue to analyze the impacts of price-gouging regulation 
whenever unique opportunities present themselves. Welfare analyses should be aware of the possibility that individuals learn about and anticipate policy effects, and such anticipation may ultimately impact the welfare calculus of the policy.

Supplementary Information The online version contains supplementary material available at https://doi.org/ 10.1007/s10603-021-09493-1.

Acknowledgements We thank two anonymous reviewers from the Journal of Consumer Policy and Professor Michael A. Giberson from Texas Tech University for providing invaluable feedback on this work. We also thank Shivam Duttapadhyay for his assistance with this research.

\section{References}

Abadie, A., \& Imbens, G. W. (2011). Bias-corrected matching estimators for average treatment effects. Journal of Business \& Economic Statistics, 29(1), 1-11.

Abadie, A., Drukker, D., Herr, J. L., \& Imbens, G. W. (2004). Implementing matching estimators for average treatment effects in stata. The Stata Journal, 4(3), 290-311.

Barzel, Y. (1974). A theory of rationing by waiting. The Journal of Law Economics, 17(1), 73-95.

Beatty, T. K. M., Lade, G. E., \& Shimshack, J. (2021). Hurricanes and gasoline price gouging. Journal of the Association of Environmental and Resource Economists, 8(2), 347-374.

Bikchandani, S., \& Hirshleifer, D. (1992). A theory of fads, fashion, custom, and cultural change as informational cascades. Journal of Political Economy, 100(5), 992-1026.

Brewer, M. (2007). Planning disaster: Price gouging statutes and the shortages they create. Brooklyn Law Review, 72(3).

CA.gov. (2020). Governor Newsom issues executive order to protect consumers from price gouging. Retrieved from gov.ca.gov/2020/04/03/governor-newsom-issues-executive-order-to-protect-consumers-from-price-gouging/. Accessed 15 June 2021.

Cabral, L., \& Xu, L. (2020). Seller reputation and price gouging: Evidence from the covid-19 pandemic. Covid Economics, 12, 1-20.

Chakraborti, R., \& Roberts, G. (2020). Anti-price gouging laws, shortages, and covid-19: Insights from consumer searches. Journal of Private Enterprise, 35(4), 1-20.

Culpepper, D., \& Block, W. E. (2008). Price gouging in the Katrina aftermath: Free markets at work. International Journal of Social Economics, 35(7), 512-520.

Deacon, R. T., \& Sonstelie, J. (1989). The welfare costs of rationing by waiting. Economic Inquiry, 27(2), 179196.

Duflo, E., Glennerster, R., \& Kremer, M. (2008). Using randomization in development economics research: A toolkit. In T. P. Schultz \& J. A. Strauss (Eds.), Handbook of development economics (Vol. 4, 1st ed., pp. 3895-3962). Elsevier.

Fleck, R. K. (2014). Can prohibitions on 'price gouging' reduce deadweight losses? International Review of Law and Economics, 37, 100-107.

Fredriksson, A., \& de Oliveira, G. M. (2019). Impact evaluation using difference-in-differences. RAUSP Management Journal, 54(4), 519-532.

Gardizy, A. (2020). We're still hoarding toilet paper because of coronavirus, and for no good reason. The Boston Globe. Retrieved from https://www.bostonglobe.com/2020/03/30/nation/were-still-hoarding-toilet-paperbecause-coronavirus-no -good-reason/. Accessed 15 June 2021.

Ghinai, I., McPherson, T. D., Hunter, J. C., Kirking, H. L., Christiansen, D., Joshi, K., Team, I. C.-. I, et al. (2020). First known person-to-person transmission of severe acute respiratory syndrome coronavirus 2 (sarscov-2) in the usa. Lancet, 395(10230), 1137-1144.

Greene, W. H. (2017). Econometric analysis (Vol. 8). Pearson.

Hansman, C., Hong, H., de Paula, A., \& Singh, V. (2020). A sticky-price view of hoarding (NBER Working Papers No. 27051). National Bureau of Economic Research, Inc. 
Hiltzik, M. (2017). Memo to economists defending price gouging in a disaster: It's still wrong, morally and economically. Los Angeles Times. Retrieved from https://www.latimes.com/business/hiltzik/la-fi-hiltzikprice-gouging-harvey-20170828-story.html. Accessed 15 June 2021.

Huremovic, D. (2019). Psychiatry of pandemics: A mental health response to infection outbreak. Springer.

Imbens, G. (2004). Nonparametric estimation of average treatment effects under exogeneity: A review. The Review of Economics and Statistics, 86(1), 4-29.

John Hopkins University \& Medicine. (2020). Coronavirus resource center. Retrieved from https:/coronavirus. jhu.edu/map.html. Accessed 15 June 2021.

King \& Spalding Law Firm. (2020). Covid-19 survey of federal and state price gouging laws. Retrieved from https://www.kslaw.com/pages/covid-19-survey-of-federal-and-state-price-gouging-laws

Lechner, M. (2011). The estimation of causal effects by difference-in-difference methods. Foundations and Trends in Econometrics, 4(3), 165-224.

Louisiana State Legislature. (2020). Rs 29:732. Retrieved from http://legis.la.gov/legis/Law.aspx?d=85680. Accessed 15 June 2021.

Montgomery, D. W., Baron, R. A., \& Weisskopf, M. K. (2007). Potential effects of proposed price gouging legislation on the cost and severity of gasoline supply interruptions. Journal of Competition Law and Economics, 3(3), 357-397.

Niles, R. (2020). To end the shortages, repeal the anti-price gouging laws. Real Clear Markets. Retrieved from https:/www.realclearmarkets.com/articles/2020/04/08/to_end_the_shortages_repeal_the_anti-price_ gouging laws 488608.html. Accessed 15 June 2021.

Pan, J. (2020). The pandemic's shameless profiteers - while hucksters and quacks try cash in on the crisis, the coronavirus is fast becoming a windfall for those already on top. The New Republic. Retrieved from https:// newrepublic.com/article/157150/coronavirus-pandemic-hucksters-alex-jones-big-pharma. Accessed 15 June 2021.

Platt, B. C. (2009). Queue-rationed equilibria with fixed costs of waiting. Economic Theory, 40(2), $247-274$.

Snyder, J. (2009). What's the matter with price gouging? Business Ethics Quarterly, 19(2), 275-293.

Sterman, J. D., \& Dogan, G. (2015). I'm not hoarding, I'm just stocking up before the hoarders get here. Journal of Operations Management, 39-40(1), 6-22.

Tarrant, M. S. (2015). The effects on anti-price gouging laws in the wake of a hurricane. (Master's thesis, Montana State University, 2015).

Weitzman, M. L. (1991). Price distortion and shortage deformation, or what happened to the soap? The American Economic Review, 81(3), 401-414.

World Health Organization. (2020). Coronavirus disease (covid-19) - events as they hap- pen. World Health Organization. Retrieved from https://www.who.int/emergencies/diseases/novel-coronavirus-2019/events-asthey-happen. Accessed 15 June 2021.

Zwolinski, M. (2008). The ethics of price gouging. Business Ethics Quarterly, 18(3), 347-378.

Publisher's Note Springer Nature remains neutral with regard to jurisdictional claims in published maps and institutional affiliations.

\section{Affiliations}

\section{R. Chakraborti ${ }^{1} \cdot$ G. Roberts $^{2}$}

1 Dept. of Economics, Christopher Newport University, 1 Avenue of the Arts, Newport News, VA 23606, USA

2 Dept. of Economics, John B. Goddard School of Business \& Economics, Weber State University, 1337 Edvalson St, Dept 3801, Ogden, UT 84408, USA 